\title{
Nonreciprocal Acoustic Transmission using Lithium Niobate Parity-Time-Symmetric Resonators
}

\author{
Linbo Shao ${ }^{1}$, Wenbo Mao ${ }^{1,2}$, Smarak Maity ${ }^{1}$, Neil Sinclair ${ }^{1,3}$, Yaowen Hu ${ }^{1}$, \\ Lan Yang ${ }^{2}$, and Marko Lončar ${ }^{1}$ \\ ${ }^{1}$ John A. Paulson School of Engineering and Applied Sciences, Harvard University, 29 Oxford Street, Cambridge, MA 02138, USA \\ ${ }^{2}$ Department of Electrical and Systems Engineering, Washington University, St. Louis, MO 63130, USA and \\ ${ }^{3}$ Division of Physics, Mathematics and Astronomy, and Alliance for Quantum Technologies (AQT), California Institute of Technology, 1200 E. \\ California Boulevard, Pasadena, CA 91125, USA \\ shaolb@seas.harvard.edu (L.S.); loncar@seas.harvard.edu (M.L.)
}

\begin{abstract}
Taking advantage of the piezoelectricity of lithium niobate, we achieve nonreciprocal transmission of 10 decibels for a $200-\mathrm{MHz}$ surface acoustic wave using parity-timesymmetric resonators and demonstrate one-way circulation of acoustic waves. @ 2020 The Author(s)
\end{abstract}

\section{Introduction}

Phonons have emerged as versatile on-chip information carriers with applications ranging from microwave filters to transducers. Nonreciprocal devices are desired to control and route these high-frequency phonons. Nonreciprocal phonon transmission can be achieved by breaking the time-reversal symmetry of propagating waves. Previous demonstrations, which employ bulk media based on circulating fluids [1] and superlattices with nonlinear media [2, 3], were limited to acoustic frequencies below a few MHz. Meanwhile, ferromagnetic materials demonstrate nonreciprocal phonon device at microwave frequencies but with a weak isolation [4]. Here, we construct a nonlinear acoustic parity-time(PT)-symmetric system and enable nonreciprocal transmission. We achieve a nonreciprocity of 10 decibels for a $200-\mathrm{MHz}$ surface acoustic wave (SAW) and further demonstrate one-way circulation of SAW by cascading nonreciprocal devices.

\section{PT-symmetric SAW resonators}

The PT-symmetric SAW system consists of two coupled SAW resonators defined by the Bragg mirrors (Fig. 1(a)). The Bragg mirrors provide more than $30 \mathrm{~dB}$ reflectivity over a bandwidth of $8 \mathrm{MHz}$ around a frequency of $200 \mathrm{MHz}$ (Fig. 1(b)). The SAW resonators exhibit three resonant modes with intrinsic quality factor up to $10^{4}$ within the reflection band of the Bragg mirror. The interdigital transducers (IDTs) within the resonator are connected to external electronic circuits to provide the gain or loss for the SAW. Specifically, the variable gain IDT is connected (using wire bonding) to a negative resistance electric circuit implemented by an operational amplifier with feedback resistors, and the loss IDT is connected to a tunable electronic resistor. Two more IDTs are situated outside the coupled resonator system and are used as an emitter and a receiver of SAWs, for measurements of acoustic transmission.

\section{Results}

We numerically simulate the nonreciprocal SAW transmission in the broken PT-symmetric region (Fig. 1(c)). The PT symmetry breaking induces a stronger localization in the active resonator for the backward propagating wave than that of the forward propagating wave. The stronger localization results in a lower gain due to the saturation and leads to a lower transmission in the backward direction.

Our PT-symmetric SAW devices operate in the unbroken or broken symmetry regimes by varying the number of Bragg mirror grooves (i.e. the coupling strength) between the two resonators. An 80-groove (30-groove) mirror is used for measurements in the nonreciprocal broken (reciprocal unbroken) PT-symmetric regime. A nonreciprocity of $10.9 \mathrm{~dB}$ is observed in the broken PT-symmetric regime (Fig. 2(a)), while a reciprocal transmission is observed in the unbroken-PT-symmetric regime (Fig. 2(b)).

Further, we demonstrate one-way SAW circulation using two nonreciprocal devices. Expected circulating behavior is observed at the resonant frequency of the device (Fig. 3). A SAW from Port 1 is preferably transmitted to Port 2 $\left(\mathrm{S}_{21}\right)$. At the resonant frequency, a $20 \mathrm{~dB}$ higher transmission is observed in the clockwise direction $\mathrm{S}_{21}$ than that in the counterclockwise direction $\mathrm{S}_{31}$ (Fig. 3(a)). Similarly, when a SAW is excited from Port 2, a 10 dB higher transmission to Port 3 is observed than that to Port 1 (Fig. 3(b)).

\section{Conclusion}

We demonstrate a compact piezoelectric platform on lithium niobate for non-Hermitian and nonreciprocal acoustics. The operating frequency can be adjusted from a hundred megahertz to a few gigahertz by geometrically scaling the design [5]. Our work would enable exploration of acoustic non-Hermitian physics and extend acoustic signal processing functionalities for next-generation wireless communication. 
(a)

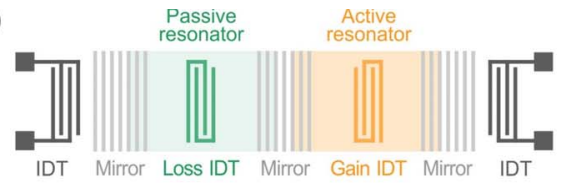

(b)

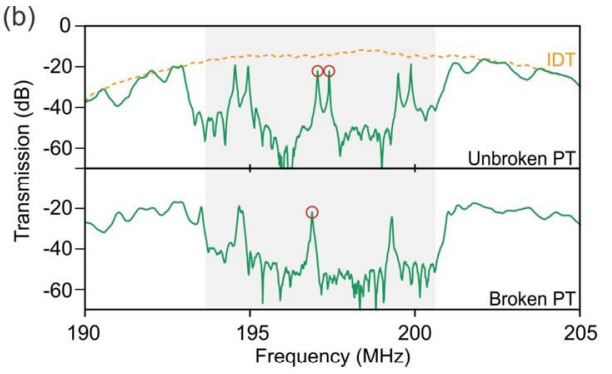

(c) Mirror Passive resonator

Forward transmission $\rightarrow$

Backward transmission $\leftarrow$

Fig. 1. Nonreciprocal phonon transmission using nonlinear PT-symmetric SAW resonators. (a) Schematic and microscopic image of our coupled SAW resonator system for nonreciprocal transmission. Loss and gain are introduced in the passive and active resonators, respectively. IDTs are used to create gain, loss and to generate and receive SAWs. (b) Measured transmission spectra of one device with a strong coupling in the unbroken PT-symmetric (reciprocal) regime and the other device with a weak coupling in the broken PT-symmetric (nonreciprocal) regime. (c) Numerical simulations of the magnitude of elastic displacement due to SAWs propagating through broken PTsymmetric (nonreciprocal) resonators in the forward and backward directions.
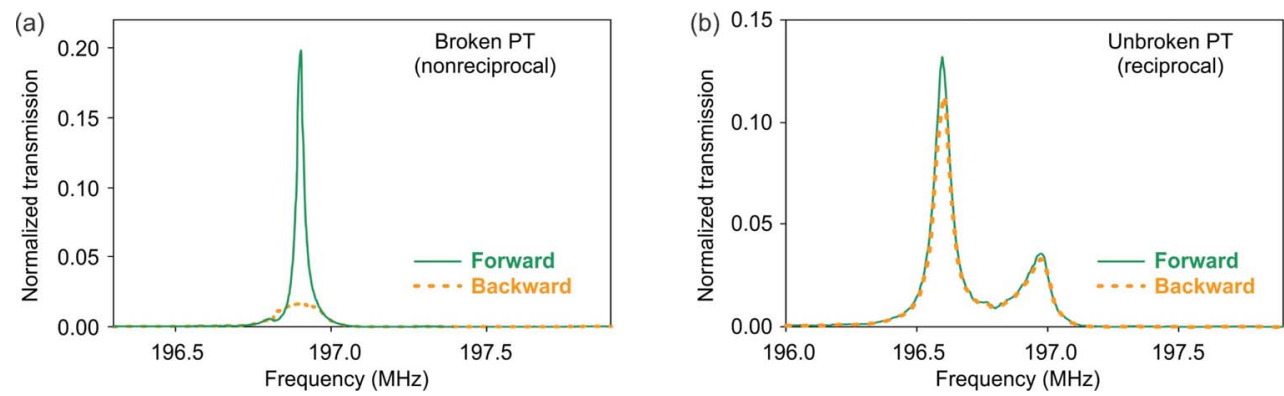

Fig. 2. (a) Nonreciprocal and (b) reciprocal transmission measurements of the SAW resonators in the broken (unbroken) PT-symmetric regime. The microwave powers applied to input IDTs are $-25 \mathrm{dBm}(3 \mu \mathrm{W})$ in both plots.
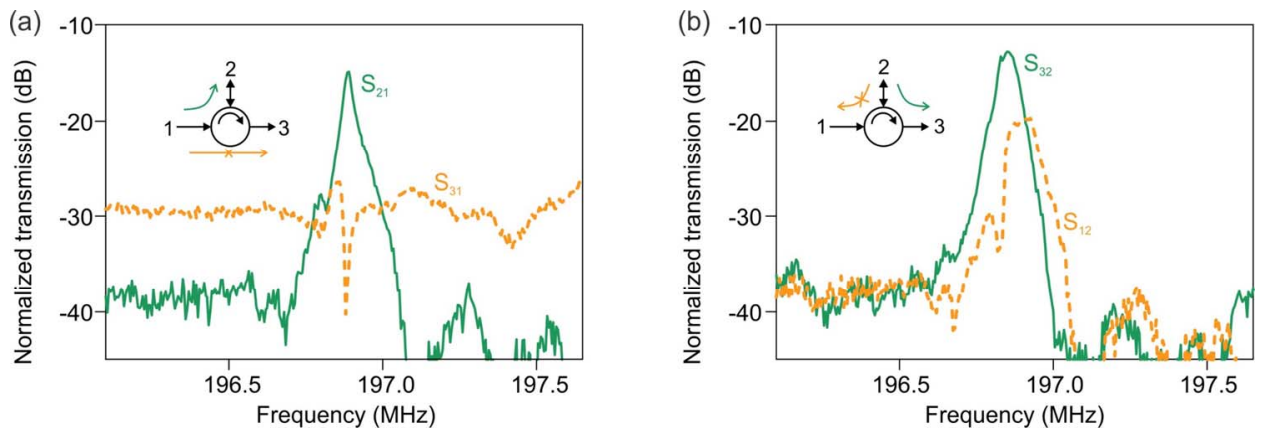

Fig. 3. One-way circulation of SAW. Transmission measurements among different ports.

\section{Acknowledgment}

This work is supported by the STC Center for Integrated Quantum Materials, NSF Grant No. DMR-1231319, NSF CQIS Grant No. ECCS-1810233, ONR MURI Grant No. N00014-15-1-2761, AFOSR MURI Grant No. FA9550-14-1-0389. N.S. acknowledges support by the Natural Sciences and Engineering Research Council of Canada (NSERC), the AQT Intelligent Quantum Networks and Technologies (INQNET) research program, and by the DOE/HEP QuantISED program grant, QCCFP (Quantum Communication Channels for Fundamental Physics), award number DESC0019219.

\section{References}

1. R. Fleury, et al., "Sound Isolation and Giant Linear Nonreciprocity in a Compact Acoustic Circulator," Science 343, 516-519 (2014).

2. B. Liang, et al., "An acoustic rectifier," Nat. Mater. 9, 989-992 (2010).

3. B. Liang, et al., "Acoustic diode: rectification of acoustic energy flux in one-dimensional systems," Phys. Rev. Lett. 103, 104301 (2009).

4. T. Nomura, et al., "Phonon Magnetochiral Effect," Phys. Rev. Lett. 122, 145901 (2019).

5. L. Shao, et al., "Phononic Band Structure Engineering for High-Q Gigahertz Surface Acoustic Wave Resonators on Lithium Niobate," Physical Review Applied 12, 014022 (2019). 